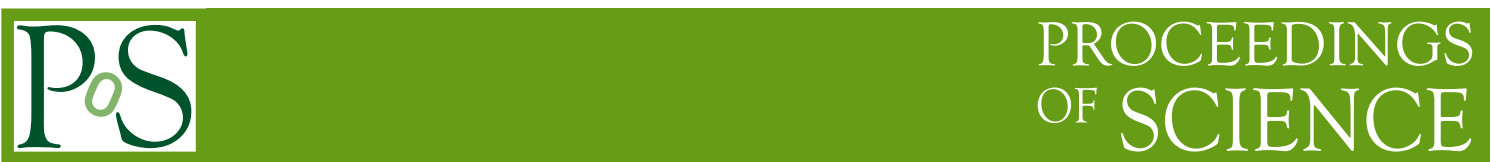

\title{
Mediterranean Neutrino Telescopes
}

\section{Paschal Coyle*}

CPPM, Aix Marseille University \& CNRS/IN2P3

E-mail: coyle@cppm.in2p3.fr

\begin{abstract}
ANTARES is a deep sea neutrino telescope located in the deep sea 40km off the southern coast of France. The results of searches for cosmic neutrinos produced in astrophysical sources and from annhilation of dark matter in the Sun and Galactic centre are presented.

The European neutrino astronomy community has recently started the construction of KM3NeT, a several cubic kilometre neutrino telescope. The R\&D for this new research infrastructure are described. Finally, the potential for a measurement of the neutrino mass hierarchy, with a densely instrumented detector configuration (ORCA) is discussed.
\end{abstract}

Frontiers of Fundamental Physics 14 - FFP14,

15-18 July 2014

Aix Marseille University (AMU) Saint-Charles Campus, Marseille

\footnotetext{
${ }^{*}$ Speaker.
} 